\title{
Participation of low molecular weight antioxidants selenium and caffeic acid in the regulation of respiration in Solanum Tuberosum in hypothermic conditions
}

\author{
Tamara Puzina ${ }^{1, *}$, Inna Makeeva ${ }^{1}$, and Pavel Prudnikov ${ }^{2}$ \\ ${ }^{1}$ Orel State University named after I.S. Turgenev, 302026, 95, Komsomolskaya str., Orel, Russian \\ Federation \\ ${ }^{2}$ All-Russian Scientific Research Institute for Breeding Fruit Crops, 302530, village Zhilina, Orel, \\ Russian Federation
}

\begin{abstract}
To study the effect of low molecular weight antioxidants on the respiration process, the content of auxins and abscisic acid, 15-day-old potato (Solanum tuberosum) tubers grown under laboratory conditions were sprayed with $5.8 \mu \mathrm{M}$ sodium selenite solution or $0.1 \mathrm{mM}$ caffeic acid solution. Control runners were treated with water. Hypothermia $\left(-2{ }^{\circ} \mathrm{C}\right)$ simulating freezing was created for 2 hours. 21-day-old runners were analyzed. Under optimal temperature conditions, the studied antioxidants stimulated the respiration process, while selenite had a greater effect. Caffeic acid increased the maintenance respiration rate. Selenite and caffeic acid increased the auxin content in the runners. Selenite reduced the amount of abscisic acid, resulting in an increase in the indoleacetic acid/abscisic acid ratio. Under hypothermic conditions, caffeic acid significantly reduced the increase in respiration, and in the option with selenite, an insignificant stabilization of this process was noted with a greater decrease in indoleacetic acid/abscisic acid ration as compared with optimal conditions.
\end{abstract}

\section{Introduction}

Breathing is the supplier of both energy and plastic ingredients to the aerobic cell. Therefore, to study the ways of its regulation under optimal and, first of all, under stressful environmental conditions is of interest. The data available in the references indicate the sensitivity of the respiration process to the treatment of plants with antioxidants. Among the low molecular weight antioxidants, researchers have recently paid attention to the microelement selenium. It is part of glutathione peroxidase, glutathione-S-transferase, which are involved in maintaining peroxide homeostasis. The effect of selenium on work of antioxidant enzymes under stressful conditions has been shown in many studies. Stabilization of stress-stimulated activity of catalase and, first of all, peroxidase in wheat plants under drought conditions under the influence of sodium biselenite is noted [1]. It was

*Corresponding author:tipuzina@gmail.com 
shown that under hyperthermia $\left(20^{\circ} \mathrm{C}\right)$ sodium selenate inhibited the activity of superoxide dismutase, but slightly increased the activity of ascorbate peroxidase in the Eastern galega [2]. In the references, there is information about its effect on different aspects of the breathing process. Therefore, activation of 3-phosphoglycerol aldehyde dehydrogenase, which is involved in glycolysis, has been shown [3]. Stimulation of succinate dehydrogenase activity by selenium at a concentration of $5 \cdot 10^{-6} \%$ was noted [4]. However, a concentration of $10^{-2} \%$ inhibited the function of this enzyme. Selenium affects the work of not only dehydrogenases involved in the respiration process, but also oxidases, namely, cytochromes. It is a good catalyst for the cytochrome $\mathrm{C}$ recovery [5]. There is also an increase in the phosphorylating activity of mitochondria in peas and corn when using a growth-stimulating dose of selenium [6]. On the contrary, use of an inhibitory dose of selenium against the background of an increase in the respiratory activity of mitochondria leads to an almost double decrease in phosphorylating activity. According to the authors, high doses of selenium uncouple the processes of oxidation and phosphorylation. In studies [7], sodium selenite significantly increased the respiration rate in the leaves and flowers of Brassica rapa grown in hydroponics. In isolated studies, the participation of selenium in the regulation of respiration under stressful environmental conditions is noted. A decrease in the respiratory potential of mitochondria in potato plants measured by the activity of electron transport under the influence of selenium in four weeks after drought has been shown [8]. Under conditions of salt stress, application of selenium to the soil $(0.2$ and $4 \mathrm{~g} / \mathrm{l})$ decreased the respiration rate, but increased the biological productivity of Brassica napus oil plants. In the references, no information was found on the effect of selenium on the respiration process under hypothermia.

At present, attention is being paid to the study of the antioxidant properties of secondary metabolites, and first of all, phenolic compounds. For a long time, it was believed that they have inhibitory properties only. Recently, however, it has been shown that phenolic compounds can stimulate growth and organ formation. The physiological and biochemical role of individual groups of these compounds has been studied to varying degrees. Antioxidant properties of flavonoids are most widely studied [9-13]. As for caffeic acid, a representative of hydroxycinnamic acids that make up phenylpropanoids, its antioxidant properties have been shown in a small number of works. Namely, in studies carried out on soy plants with addition of $100 \mu \mathrm{M}$ caffeic acid solution to the nutrient medium, a significant decrease in the content of superoxide anion radical was noted due to an increase in the activity of manganese and copper/zinc isoforms of superoxide dismutase [14]. Similar data on the content of superoxide radical anion and superoxide dismutase activity were obtained on cucumber plants, while stimulation of the activity of catalase, peroxidase, glutathione and ascorbate peroxidase, as well as a tendency to a slight increase in the content of low molecular weight antioxidant proline, and, as a consequence, a decrease in malondialdehyde $25 \mu \mathrm{M}$ caffeic acid solution [15].

There is practically no information on the effect of caffeic acid on the respiration process. Only in a study [9], it was shown that caffeic acid in isolated mitochondria of corn and cucumber, unlike other phenolic compounds, is not an uncoupler of the process of oxidation and phosphorylation. In domestic papers using the Zircon preparation synthesized on the basis of a mixture of hydroxycinnamic acids, which include caffeic acid, there is no effect on the intensity of the respiration process in wheat plants under optimal environmental conditions and a slight decrease under the influence of drought [16]. In a study [17] carried out on timothy plants, respiration was inhibited under the action of a number of temperatures $\left(10-40^{\circ} \mathrm{C}\right)$ when seeds were treated with a $0.001 \mathrm{M}$ solution of Zircon. 
At present, the physiological mechanism of action of the antioxidants selenium and caffeic acid on the respiration process in a plant body, both under optimal and stressful environmental conditions, remains practically unstudied.

The aim of the study was to reveal the effect of selenite and caffeic acid on the respiration rate of the runners of potato tubers recovery under optimal conditions and under the action of hypothermia $\left(-2{ }^{\circ} \mathrm{C}\right)$. Wherein, attention was paid to the influence of these antioxidants on the content and ratio of phytohormones.

\section{Methods}

The object of the study was the 21-day-old runners of recovery of potato tubers (Solanum tuberosum L.), Zhukovsky variety, early selection of Federal State Funded Research Institution Federal Potato Research Center named after A.G. Lorkha (Korenevo, Russia).

Laboratory experiments were carried out under the conditions of the laboratory "Mechanisms of Regulation of Plant Growth and Development" of Orel State University named after I.S. Turgenev. To obtain 21-day-old runners of recovery, potato tubers that had emerged from a state of deep dormancy were transferred from a vegetable store, where they were stored at a temperature of $+4{ }^{\circ} \mathrm{C}$, to laboratory conditions and germinated in containers with wet sawdust at the beginning in the dark, and after the emergence of runners on the surface of the substrate at a temperature of $20 \pm 2{ }^{\circ} \mathrm{C}$ and 14 hours of illumination.

Runners were treated with $5.8 \mu \mathrm{M}$ sodium selenite solution or $0.1 \mathrm{mM}$ caffeic acid solution (Sigma, United States) by spraying 15 days after germination. Control runners were sprayed with water. Hypothermia was created by placing containers with tuber recovery runners in a low-temperature cabinet $\mathrm{T}-25 / 01$ (Russia) at a temperature of $-2{ }^{\circ} \mathrm{C}$, simulating frosts, which have a detrimental effect on Solanum tuberosum.

Respiration intensity was determined by titration by the amount of $\mathrm{CO}_{2}$ evolved in the gas exchange vessels (Fizpribor, Russia). A weighed portion of the plant material was placed on the grid of the device, and $0.1 \mathrm{~N} \mathrm{Ba}(\mathrm{OH})_{2}$ was added to the lower part. The vessel was hermetically sealed. After 15 minutes of exposure, $\mathrm{Ba}(\mathrm{OH})_{2}$ was titrated with $0.1 \mathrm{~N} \mathrm{HCl}$ preliminarily adding phenolphthalein. An option without a plant sample was used as a control. Respiration rate was expressed as $\mathrm{mg}$ of $\mathrm{CO}_{2} /(\mathrm{h} g$ wet weight).

The maintenance breath was determined by placing the test plants in the dark for 48 hours. The speed of $\mathrm{CO}_{2}$ release after plant being in the dark helped to obtain the value of maintainance respiration intensity [18]. Growth respiration was calculated from the difference between plant respiration in the light and after keeping them in the dark.

Extraction of auxins and abscisic acid was carried out with $80 \%$ ethanol from one sample of recovery runners preliminarily fixed with liquid nitrogen. Extraction purification of hormones was carried out according to the method of $\mathrm{S}$.Yu. Veselov [19]. After evaporation of the extract at IR-1M rotary evaporator (Russia) up to an aqueous residue, after acidification ( $\mathrm{pH} 2-3$ ), indoleacetic acid and abscisic acid were extracted twice with diethyl ether. Acid auxins and abscisic acid were reextracted from the combined organic phase with $1 \%$ sodium bicarbonate in a 1:3 ratio. The organic phase was separated and discarded, and auxins and abscisic acid were recovered twice from the aqueous phase (after acidification up to $\mathrm{pH} 2-3$ ) with diethyl ether and methylated with diazomethane. The content of phytohormones (indoleacetic and abscisic acids) was determined by the method of enzyme-linked immunosorbent assay [20] using reagents (Uralinvest, Ufa). The dry residues of the hormone extracts were dissolved in ethanol and analyzed in the wells of a polystyrene plate. After sorption of the protein conjugate of the hormone, rabbit serum with antibodies to the corresponding hormone, and then a solution of a standard hormone or experimental samples was added to the wells. The amount of antibodies specifically bound 
to the protein conjugate of the hormone sorbed in the wells was determined using mutton antibodies against rabbit immunoglobulins labeled with peroxidase. Orthophenylenediamine was used to determine the activity of bound peroxidase. The intensity of the chromophore response (color intensity) was determined on a Dombiplate microphotometer (Dia - M, Russia) at a wavelength of $492 \mathrm{~nm}$. Indoleacetic acid and abscisic acid (Serva, Germany) were taken as standard solutions of phytohormones.

The figures show the arithmetic means of five biological replicates and their standard errors. Analytical replication is fivefold. The reliability of the results was assessed using the Student's criterion considering the differences to be significant at a confidence level above 0.95. Statistical analysis of the data was carried out in MS Excel spreadsheets and Statistica 6.1 software.

\section{Results}

Determination of the respiration intensity in 21-day-old recovery runners revealed an increase in this process under the influence of the studied antioxidants (Fig. 1), and sodium selenite had a greater effect compared to caffeic acid. Therefore, the stimulation in the option with selenite-ion was $39 \%$, while in the option with caffeic acid it was $23 \%$. An increase in respiration in corn and peas under the influence of sodium selenate was also found in [6]. As for caffeic acid, studies [16] carried out on wheat plants did not reveal a change in respiration when treated with Zircon, which contains caffeic acid.

It is known that the nonspecific response to stress is the activation of the respiration process. Indeed, the results obtained showed that under conditions of 2-hour hypothermia ($2{ }^{\circ} \mathrm{C}$ ), the respiration intensity in the control runners increased sharply (by 2.5 times). Almost the same effect was noted under the influence of selenite-ion. Under these conditions, caffeic acid slightly (by $22 \%$ ) increased the intensity of respiration. As a result, caffeic acid stabilized the breathing process under stress. Its intensity decreased by $40 \%$ versus control. Wherein, in the option with selenite, respiration increased by $21 \%$, which is less in comparison with optimal conditions. This may indicate some stabilization of this process under the influence of selenium.

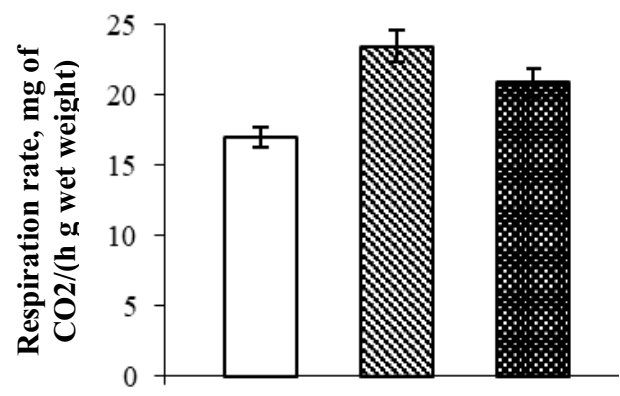

Optimal temperature

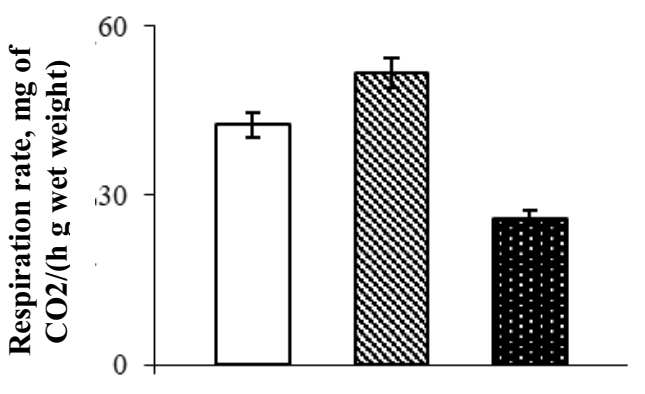

2-hour hypothermia

$\square$ - control, $\mathbf{\square}-\mathrm{Na}_{2} \mathrm{SeO}_{3}, \mathbf{\square}$ - caffeic acid

Fig. 1. Influence of sodium selenite and caffeic acid on the respiration rate of runners under optimal temperature conditions and under hypothermia.

Along with the general respiration rate, the functional components of this process were studied, namely, maintenance respiration and growth respiration, which characterize the expenditure of respiration energy to maintain the existing plant biomass and the energy spent on the new growth processes [21]. The proportion of respiration to maintain the recovery runners in the control option was $70 \%$ in the total respiration, while in runners 
enriched with caffeic acid it was $80 \%$ (Fig. 2). Apparently, this is due to the fact that the treated plants had a large biomass of runners. So, 7 days after spraying with caffeic acid, their dry weight was $28 \%$ more than in the control.
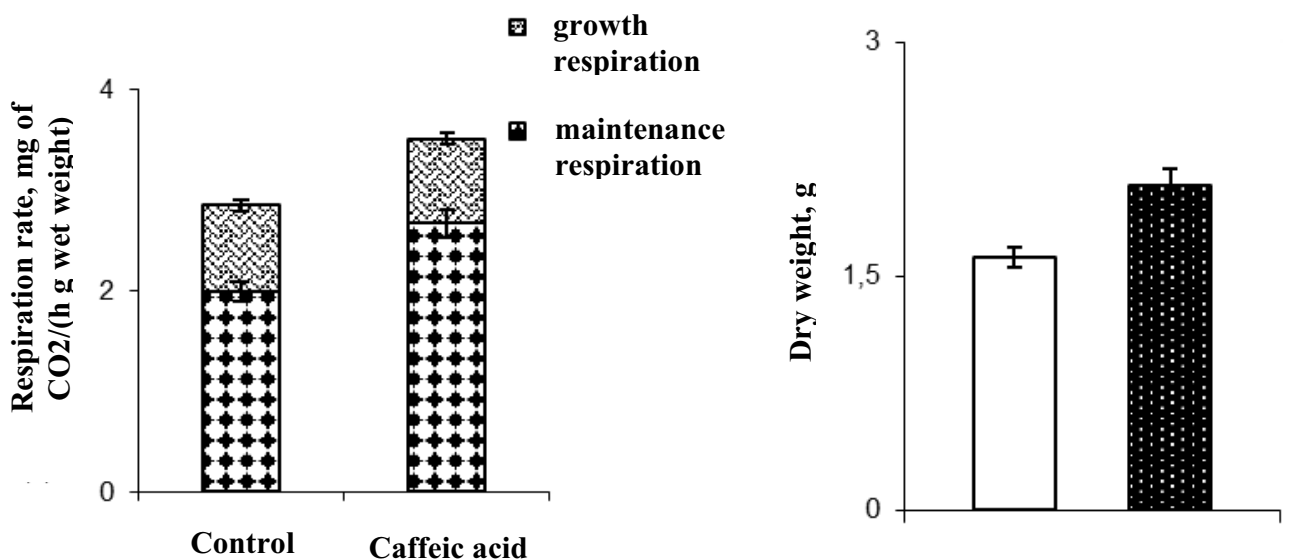

Caffeic acid

$\square$ - control, $\square$ - caffeic acid

Fig. 2. Influence of caffeic acid on the functional components of the respiration process and the mass of runners.

To elucidate the physiological mechanism of action of selenite and caffeic acid on the respiration process, their influence on the hormonal status of the recovery runners was studied. As can be seen from the data shown in Fig. 3, under optimal conditions, the studied antioxidants increased the content of auxins: sodium selenite - by $78 \%$, while caffeic acid by $-36 \%$.
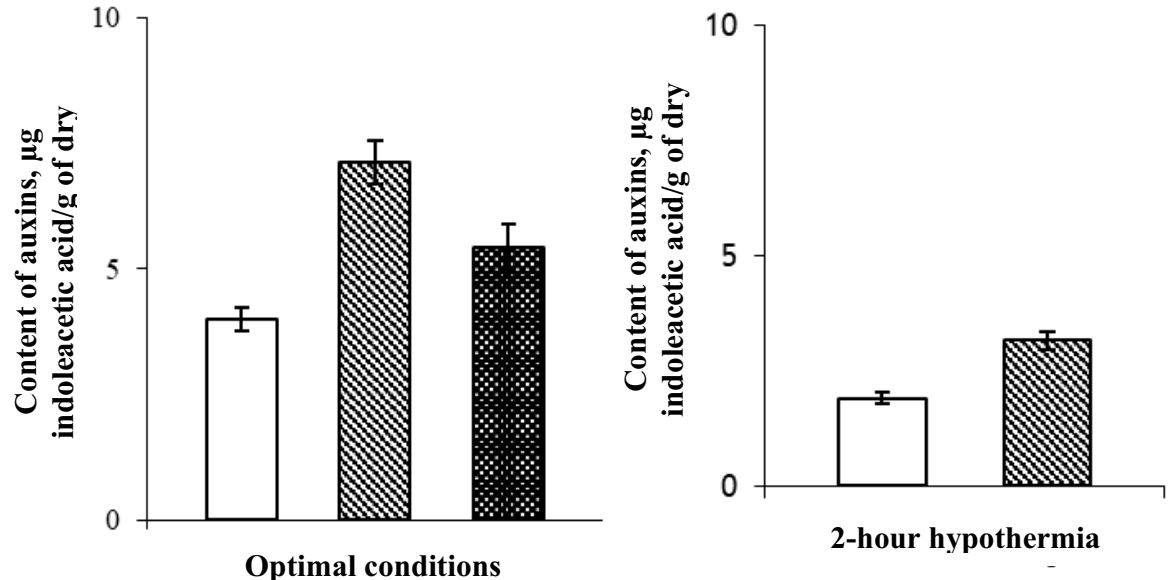

$\square$ - control, $\mathbf{\square}-\mathrm{Na}_{2} \mathrm{SeO}_{3}, \mathbf{\square}$ - caffeic acid

Fig. 3. Influence of sodium selenite and caffeic acid on the content of auxins in runners.

The effect of hypothermia sharply reduced the content of endogenous auxins (2 times) in the runners of the control option and those enriched with the antioxidant selenium. 
Wherein, the level of indoleacetic acid under the influence of selenium, and in this case, significantly exceeded the control.

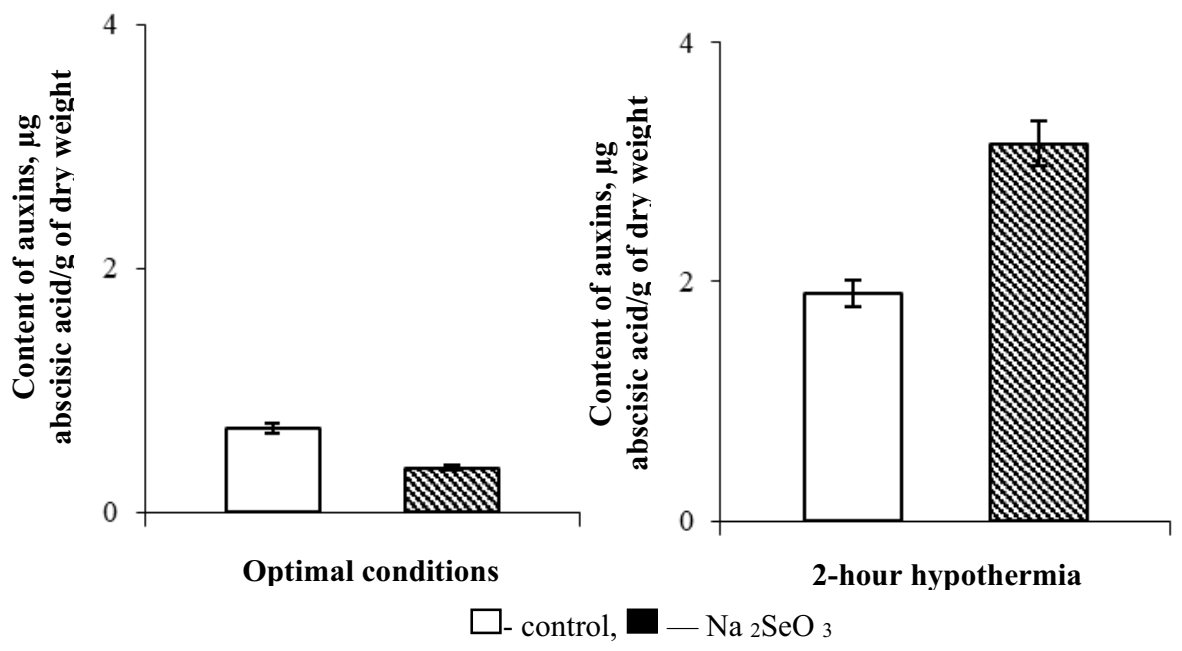

Fig. 4. Influence of sodium selenite on the content of abscisic acid in runners.

In contrast to auxins, selenite-ion reduced the content of abscisic acid by almost two times under optimal temperature conditions (Fig. 4). As a result, the indoleacetic acid/abscisic acid ratio was 19 versus 6 in the control. Keeping runners for two hours at $2{ }^{\circ} \mathrm{C}$ increased the abscisic acid content and reduced the indoleacetic acid/abscisic acid ratio under the influence of selenite - to 3.9 , and in the control — to 0.9 .

\section{Discussion}

In most studies, attention is paid to the study of the effect of a high-molecular antioxidant system on physiological and biochemical processes in a plant organism under the action of stressful environmental factors. The effect of low molecular weight antioxidants has been studied to a lesser extent. First of all, this concerns secondary metabolites, namely phenylpropanoids, which include caffeic acid. Earlier, we [22], as well as Polish and Chinese researchers, have shown that caffeic acid has antioxidant properties in plants [14, 15]. As the results of our work have shown, the respiration process is sensitive to the treatment of plants with selenium and caffeic acid. Wherein, the specificity of their action was revealed. Thus, selenite-ion enhanced respiration both under optimal and stressful temperature conditions $\left(-2{ }^{\circ} \mathrm{C}\right)$, which had a detrimental effect on potato plants. While caffeic acid stabilized this process in hypothermia.

Until now, the physiological mechanism of action of antioxidants is poorly understood. Therefore, their participation in the breathing process remains open. Meanwhile, it is known that the respiration process is regulated by phytohormones [23-25]. Determination of the content of endogenous auxins in the runners of recovery of tubers revealed a significant increase in this group of hormones, especially under the influence of selenite. This effect of selenium, according to [26], may be associated with inhibition of the activity of the auxin metabolism enzyme indoleacetic acid oxidase. Under optimal conditions, selenite reduced the level of endogenous abscisic acid and increased the indoleacetic acid/abscisic acid ratio. Accumulation of indoleacetic acid under the influence of caffeic 
acid is apparently due to the protective role of hydroxycinnamic acids (caffeic, ferulic, synapic) in the oxidation of auxins. Such information is available in the references [27]. Increase in respiration under the influence of the studied antioxidants under optimal conditions may be associated with the regulation of the work of peroxidases, which, along with peroxidase, also have an oxidase function. We have previously shown that selenite and caffeic acid increase the activity of peroxidase [22]. Some stabilization of respiration intensity under hypothermia was observed against the background of a significant increase in the abscisic acid content and a decrease in the indoleacetic acid/abscisic acid ratio. Studies [25] also revealed inhibition of respiration in Daucus carola under the influence of exogenous abscisic acid. In the process of breathing, it is important to know the ratio of its functional components [21]. The results obtained by us indicate an increase in the proportion of respiration maintaining a greater mass of recovery runners under the influence of caffeic acid.

\section{Conclusion}

Therefore, the results of this paper allow us to conclude that, under optimal temperature conditions, the intensification of the respiration process under the influence of selenite and caffeic acid occurred against the background of an increase in the content of indoleacetic acid. The effect of selenium on respiration is associated not only with the regulation of auxin metabolism, but also with decrease in abscisic acid. Caffeic acid in the functional component of respiration increased maintenance respiration without altering growth respiration. Under conditions of hypothermia, some stabilization of the respiration process under the influence of selenite was observed with a greater decrease in the indoleacetic acid/abscisic acid ratio in comparison with the optimal temperature conditions. Under stress conditions, the stabilizing effect of caffeic acid on respiration was manifested to a greater extent against selenite.

\section{References}

1. C. C. Kuznetsov (Autoref. dis. ... cand. biol. nauk, Moscow, Moscow Agricultural Academy, 2004)

2. V.A.Vikhreva, T.I.Balakhnina, V.K.Gins, A.F. Blinokhvatov, The production process of agricultural crops, 91, 2 (2001)

3. M.Ya.Lovkova, O.V.Shulepova, S.M. Sokolova, Proceedings of the Russian Academy of Sciences (Biological Series), 6, 833 (1993)

4. Z. I. Deryabina, Proceedings of the Eighth Scientific Conference on Pharmacology, 72 (1963)

5. H.E. Gantbe, Carcinogenesis, 20, 9 (1999)

6. S.M.Fatalieva, N.V. Guzhova, Agricultural biology, 5, 782 (1978)

7. G. H. Lyons, Y. Y. Genc, K. K. Soole, J. C. R. Stangoulis, F. Liu, R. D. Graham, PLANT AND SOIL, 318, 73 (2009)

8. M. Germ, I. Kreft, V. Stibilj, O. Urbanc-Bericic, PLANT PHYSIOLOGY AND BIOCHEMISTRY, 45, 162 (2007)

9. M.N. Prometov, Phenolic compounds and their role in plant life, 56th Timiryazev reading, 272 (2009)

10. O.M. Andersen, K.R. Markham, Flavonoids: chemistry, biochemistry and application (CRC Press, 2005) 
11. C. Brunetti, M. di Ferdinando, F. Ferrini, M. Tattini, Plant Signal. Behav., 6, 709 (2011)

12. V. Cheynier, G. Comte, K.M. Davis, V. Lattanzio, S. Martens, Plant Physiology and Biochemistry,72, 1 (2013)

13. O.A. Makarenko, A.P. Levitsky, Physiology and biochemistry of cultivated plants, 45, 2 (2013)

14. A. Klein, M. Keyster, N. Ludidi, Acta Physiologiae Plantarum, 35, 10 (2013)

15. Y.Y. Wan, Y. Zhang, L. Zhang, Z.Q. Zhou, X. Li, Q. Shi, X. J. Wang, J.G. Bai, Acta Physiologiae Plantarum, 37, 1 (2015)

16. I.I. Seregina, A.V. Sivashova, Bulletin of the Mordovian University, 144, 2 (2008)

17. L. V. Timeyko, E. S. Kholoptseva, Modern problems and strategy of development of agricultural science in the European North of Russia, 32 (2015)

18. T.K. Golovko, Plant respiration (physiological aspects) (S., Nauka, 1999)

19. S.Y. Veselov (Ufa, BSU,1998)

20. S.Y.Veselov, G.R. Kudoyarova (Ufa, UO OF THE USSR Academy OF Sciences, 1990)

21. O.A. Semikhatova, Plant physiology, 42, 2 (1995)

22. T.I.Puzina, I.Y. Makeeva, N.S. Vlasova, Fundamental and applied problems of modern plant biology: a collection of materials of the All-Russian Scientific Conference with international participation dedicated to the 125th anniversary of the Timiryazev Institute of Plant Physiology of the Russian Academy of Sciences (Moscow, IFR RAS, 2015)

23. N.I.Yakushkina, I.A. Kulakova, On the mechanism of action of auxin, Plant growth and its regulation (MOPI, 1984)

24. R.N. Arteca, Plant Growth Substances: Principles and Applications (New York, Chapman \& Hall, 1996)

25. F.A.A.Tetteroo, R.J. Bino, J.H.W. Bergervoetand B.Hasenack. Physiologia Plantarum, 95, 154 (1995)

26. S.C. Kwid; B. Faraji; O.A. Levander, J. Biochemistry, 3, 305 (1989)

27. V.I. Baranov, Plant Physiology, 26, 7 (1979) 